\title{
Spectral weight of doping-induced states in the two-dimensional Hubbard model
}

\author{
Ansgar Liebsch \\ Institut für Festkörperforschung, Forschungszentrum Jülich, 52425 Jülich, Germany \\ (Received 8 April 2010; revised manuscript received 20 May 2010; published 29 June 2010)
}

\begin{abstract}
The spectral weight of states induced in the Mott gap via hole doping in the two-dimensional Hubbard model is studied within cluster dynamical mean-field theory combined with finite-temperature exact diagonalization. If the cutoff energy is chosen to lie just below the upper Hubbard band, the integrated weight per spin is shown to satisfy $W_{+}(\delta) \geq \delta$ ( $\delta$ denotes the total number of holes), in agreement with model predictions by Eskes et al. [Phys. Rev. Lett. 67, 1035 (1991)]. However, if the cutoff energy is chosen to lie in the range of the pseudogap, $W_{+}(\delta)$ remains much smaller than $\delta$ and approximately saturates near $\delta \approx 0.2, \ldots, 0.3$. The analysis of recent $\mathrm{x}$-ray absorption spectroscopy data therefore depends crucially on the appropriate definition of the integration window.
\end{abstract}

DOI: $10.1103 /$ PhysRevB.81.235133

PACS number(s): 71.10.Fd

\section{INTRODUCTION}

The two-dimensional single-band Hubbard model has been widely used to study the role of Coulomb correlations in the high- $T_{c}$ cuprates. One of the remarkable features of this model was pointed out long ago by Eskes et al. ${ }^{1}$ namely, that doping the system with $\delta$ holes does not yield unoccupied low-energy states of weight $\delta / 2$ per spin, like in an ordinary band insulator. Instead, as a result of strong local Coulomb interactions, this weight is approximately given by $W_{+}(\delta) \geq \delta$. The physical reason for this feature is that both lower and upper Hubbard bands must contribute to the generation of itinerant low-energy states when holes are added to the system.

In striking contrast to this prediction, recent $\mathrm{x}$-ray absorption spectroscopy (XAS) data on $\mathrm{Tl}_{2} \mathrm{Ba}_{2} \mathrm{CuO}_{6-\delta}$ and $\mathrm{La}_{2-x} \mathrm{Sr}_{x} \mathrm{CuO}_{4 \pm \delta}$ by Peets et al. ${ }^{2}$ show a linear behavior $W_{+}(\delta) \approx \delta$ only up to about $\delta \approx 0.2$. At doping concentrations in the range $\delta=0.2, \ldots, 0.3, W_{+}(\delta)$ levels off, suggesting the inapplicability of the single-band Hubbard model for these high- $T_{c}$ compounds.

Since the calculations by Eskes et al. ${ }^{1}$ were carried out for small one-dimensional clusters, it is not entirely clear to what extent the discrepancies with respect to the data in Ref. 2 might be related to the simplicity of the theoretical model. In fact, Phillips and Jarrell $^{3}$ recently claimed that state-ofthe-art many-body calculations based on the dynamical cluster approximation (DCA) (Refs. 4 and 5) do indeed predict a saturation of $W_{+}(\delta)$ close to $\delta \approx 0.2$, in agreement with the measurements by Peets et al.

The aim of this work is to demonstrate that the doping variation of the spectral weight of the induced low-energy states depends crucially on the choice of the upper limit of the energy window in which these states are counted. ${ }^{6}$ To evaluate $W_{+}(\delta)$ for the two-dimensional Hubbard model we use the cluster extension of dynamical mean-field theory (DMFT) (Refs. 7 and 8) combined with finite-temperature exact diagonalization (ED). ${ }^{9}$ The results show that, if the integration window of $W_{+}(\delta)$ is chosen to reach up to the lower edge of the upper Hubbard band, then $W_{+}(\delta) \geq \delta$, just as predicted by Eskes et al. ${ }^{1}$ If the cutoff energy, however, is chosen to lie in the range of the pseudogap, then $W_{+}(\delta)$ is much smaller than $\delta$ and approximately saturates near $\delta$ $\approx 0.2, \ldots, 0.3$, as found in Ref. 3 It is clear, therefore, that the interpretation of the XAS measurements must be based on the correct choice of the energy window over which the induced low-energy states are taken into account.

\section{RESULTS AND DISCUSSION}

The cluster DMFT calculations are carried out for the two-dimensional Hubbard model with nearest and nextnearest hopping parameters $t=0.25 \mathrm{eV}$ and $t^{\prime}=-0.075 \mathrm{eV}$, respectively (bandwidth $W=2 \mathrm{eV}$ ). The onsite Coulomb interaction is $U=2.5 \mathrm{eV}$ and the temperature is $T=0.01 \mathrm{eV}$. With this choice of parameters the system is a Mott insulator in the zero doping limit. To account for intersite correlations, the square lattice is viewed as a superlattice consisting of 2 $\times 2$ clusters. Details of these finite-temperature ED/DMFT calculations can be found in Ref. 9.

Figure 1 shows the spectral distributions for a series of hole doping concentrations. For simplicity, we show the ED cluster spectra as they can be evaluated directly at real $\omega$ without requiring analytical continuation. Since we are primarily concerned here with integrated sections of the density of states, these cluster spectra are adequate for our analysis. The electron density per spin is given by $n_{\sigma}=0.5(1-\delta)$. At all dopings, the upper Hubbard band is seen to be separated from the low-energy states by a broad minimum related to the Mott gap in the half-filled limit. The lower edge of this band gradually shifts from $\omega=1.5 \mathrm{eV}$ at low doping to about $3.0 \mathrm{eV}$ at large doping. The low-energy states close to $E_{F}$ $=0$ reveal a pseudogap at about $50 \mathrm{meV}$ for doping up to about 0.1. In Ref. 9 we showed that the origin of this pseudogap can be traced back to a prominent collective mode in the imaginary part of the $(\pi, 0)$ component of the self-energy. (See also Ref. 5 and other theoretical work cited in Ref. 9.) This mode is therefore directly linked to spatial fluctuations within the $2 \times 2$ clusters and cannot be described within single-site DMFT. Electron-addition states in the vicinity of this collective mode are highly damped, giving rise to a pseudogap. In fact, the energy and strength of this mode exhibit a clear dispersion with doping, which translates into a corresponding doping variation of the mean position and 

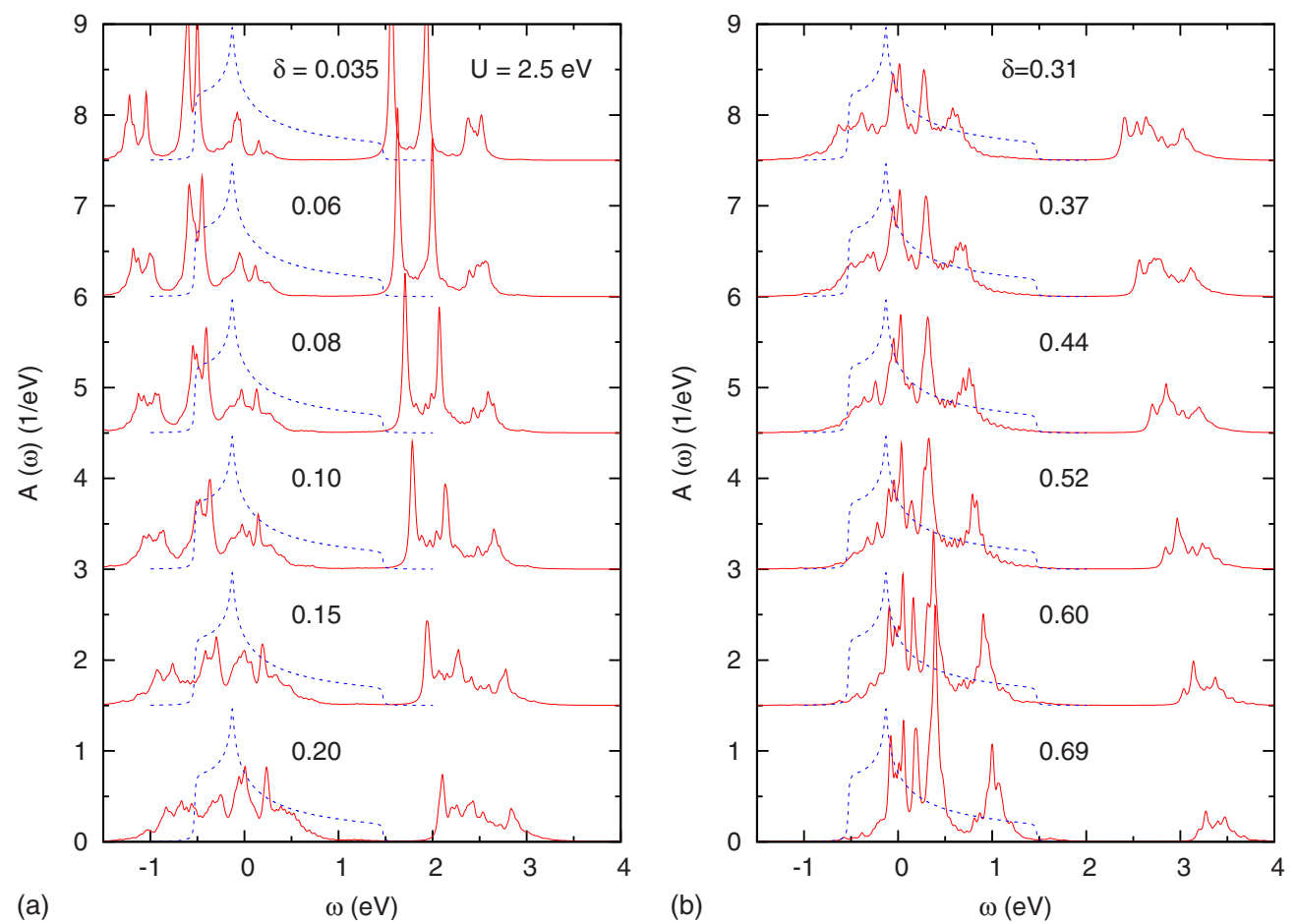

FIG. 1. (Color online) Spectral distributions calculated within cluster DMFT for two-dimensional Hubbard model at several hole dopings (broadening $\gamma=0.02 \mathrm{eV}, U=2.5 \mathrm{eV}$, and $T=0.01 \mathrm{eV}$ ). (a) $\delta=0.035, \ldots, 0.20$ and (b) $\delta=0.31, \ldots, 0.69$. The dashed lines denote the bare density of states. The pseudogap is located about $50 \mathrm{meV}$ above $E_{F}=0$ at low doping up to $\delta \approx 0.1$. The lower edge of the upper Hubbard band shifts from about $1.5 \mathrm{eV}$ at low doping to $3 \mathrm{eV}$ at large doping.

width of the pseudogap. Moreover, since the collective mode is located slightly above $E_{F}$, states in this energy range have a much shorter lifetime than states below $E_{F}$, giving rise to a pronounced particle-hole asymmetry. As shown in Fig. 2, the low-energy region of the DMFT lattice spectra, obtained via analytical continuation of the cluster self-energy to real $\omega$, is fully compatible with the cluster spectra given here in Fig. 1. (For details of the extrapolation procedure, see Ref. 9.) These lattice spectra are also qualitatively consistent with the corresponding DCA spectra for 16-site clusters (using $t^{\prime}=0$ ) derived in Ref. 3

The spectra in Figs. 1 and 2 demonstrate that the lowenergy states induced via hole doping are concentrated near $E_{F}$ only at very low doping. With increasing doping, these

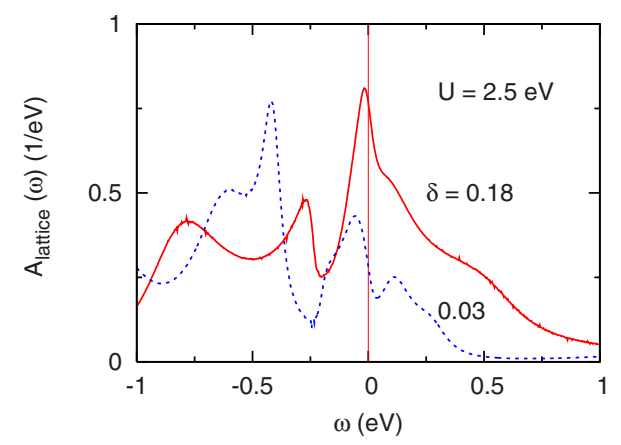

FIG. 2. (Color online) Lattice spectra for $U=2.5 \mathrm{eV}$ and $T$ $=0.01 \mathrm{eV}$ obtained via extrapolation to real $\omega$. Solid curve: hole doping $\delta=0.18$ with peak at $E_{F}=0$. Dashed curve: $\delta=0.03$ exhibiting a pseudogap above $E_{F}$. (From Ref. 9.) states spread over a larger energy window, until at unit doping an uncorrelated empty band appears between $E_{F}$ and the shifted upper band edge at $2 \mathrm{eV}$. Evidently, upon hole doping, spectral weight transfer from the upper Hubbard band begins to states near $E_{F}$ and gradually extends to the entire range up to $E_{F}+W$.

Figure 3(a) shows the integrated spectral weight of the doping-induced low-energy states, $W_{+}(\delta)$, where the upper edge $\omega_{c}$ of the energy window lies in the broad minimum below the upper Hubbard band. In the present case a convenient choice is $\omega_{c}=(1+\delta) \mathrm{eV}$. (The low-doping behavior of $W_{+}(\delta)$ was previously shown in Fig. 5 of Ref. 9. See also Ref. 10.) The results demonstrate that $W_{+}(\delta) \geq \delta$ in the entire doping range, consistent with the predictions by Eskes et al. ${ }^{1}$

Figure 3(b) shows the integrated spectral weight for a variety of fixed, doping-independent cutoff energies. For $\omega_{c}$ in the range of the pseudogap, i.e., $\omega_{c} \approx 0.2, \ldots, 0.3 \mathrm{eV}$, $W_{+}(\delta)$ remains much smaller than $\delta$ and approximately saturates near $\delta \approx 0.2, \ldots, 0.3$. The reason for this saturation is that, as pointed out above, spectral weight transfer from the upper Hubbard band must proceed at larger doping progressively to states farther above $E_{F}$, eventually covering the range up to $E_{F}+W$. For $\omega_{c} \approx 1.0, \ldots, 1.5 \mathrm{eV}, W_{+}(\delta) \geq \delta$, in agreement with Fig. 3(a). For $\omega_{c}>1.5 \mathrm{eV}$, the energy window at low doping includes part of the upper Hubbard band (see Fig. 1) so that $W_{+}(\delta)$ does not reach zero in the small doping limit. In the limit $\omega_{c} \gg 0$ (not shown), $W_{+}(\delta)$ includes all unoccupied states. Thus, $W_{+}(\delta)=1-n_{\sigma}=0.5(1+\delta)$. To capture properly the integrated spectral weight of the dopinginduced low-energy states it is evidently necessary to adjust 

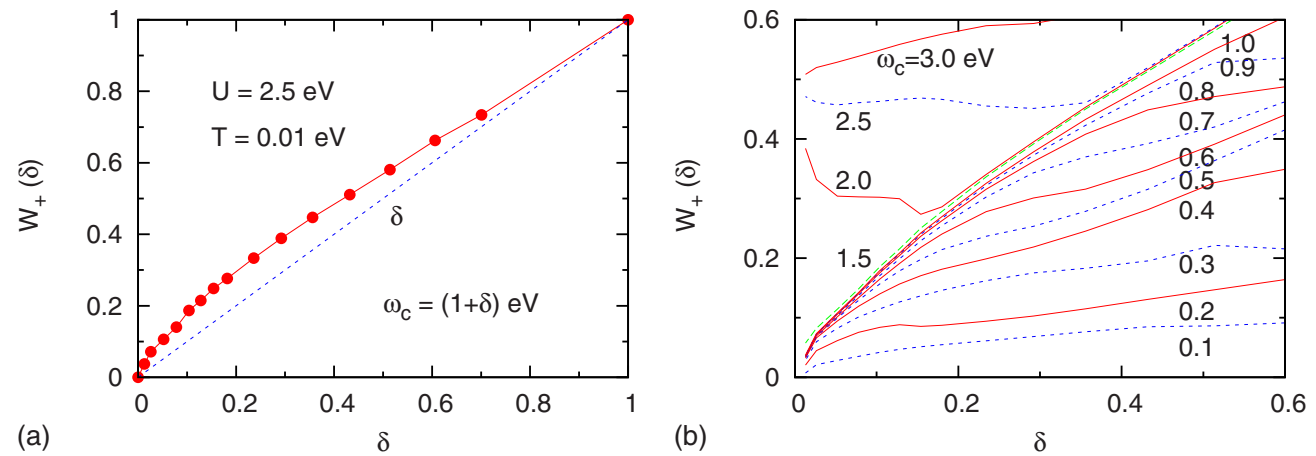

FIG. 3. (Color online) Integrated spectral weight of low-energy states per spin as a function of hole doping. (a) Integration window extends to minimum below the upper Hubbard band, with doping-dependent cutoff energy $\omega_{c} \approx(1+\delta) \mathrm{eV}$. (b) Integration window extends up to doping-independent cutoff energy $\omega_{c}=0.1 \ldots 3.0 \mathrm{eV}$.

the cutoff energy so that it roughly tracks the lower edge of the upper Hubbard band.

The cluster DMFT results discussed above are for $U$ $=2.5 \mathrm{eV}$. As can be seen in Fig. 1, the upper Hubbard band is separated from the itinerant unoccupied states by a rather wide gap of about $1, \ldots, 2 \mathrm{eV}$, regardless of doping. The low-energy spectral peak can therefore be defined without ambiguity. Since there is some uncertainty concerning the appropriate value of $U$ for the high- $T_{c}$ cuprates, we have calculated the spectral distributions also for $U=1.5 \mathrm{eV}$. As shown in Fig. 4(a), the upper Hubbard band then lies about 1 $\mathrm{eV}$ lower and overlaps the upper part of the uncorrelated density of states. The distinction between localized and itinerant states is therefore less clear than for $U=2.5 \mathrm{eV}$. Nonetheless, the high-energy peak is still separated from the lowenergy region by a shallow minimum, except in the limit of small doping. At this Coulomb energy, the system is barely insulating at half filling so that the separation between itinerant and localized states at small doping becomes highly ambiguous.

Figure 4(b) shows the integrated weight of the low-energy feature, where the minimum close to the lower edge of the upper Hubbard band is used as cutoff energy, i.e., $\omega_{c}$ $\approx 0.7, \ldots, 1.4 \mathrm{eV}$ for $\delta=0.035, \ldots, 0.32$. Because of the uncertainties pointed out above, $W_{+}(\delta)$ does not approach zero in the low-doping limit. Nevertheless, $W_{+}(\delta) \geq \delta$, in agreement with the prediction by Eskes et al. ${ }^{1}$ Compared to the analogous spectral peak for $U=2.5 \mathrm{eV}, W_{+}(\delta)$ is slightly larger for $U=1.5 \mathrm{eV}$. This trend is also consistent with the results discussed in Ref. 1 . The reason for this enhancement is the less clear distinction between itinerant and localized unoccupied states for smaller $U$, i.e., some of the incoherent weight spreads to lower energies. In spite of these uncertainties, it is evident that $W_{+}(\delta)$ does not exhibit saturation.

The experimental spectra by Peets et al. ${ }^{2}$ reveal two fairly clearly separated regions: a low-energy feature and a peak at about $1.0, \ldots, 1.5 \mathrm{eV}$ higher energy. ${ }^{11}$ It seems plausible therefore to associate the former with the doping-induced low-energy states and the latter with the upper Hubbard band, in qualitative correspondence with the spectra shown in Fig. 1. Since the lower peak has an intrinsic width of about $1 \mathrm{eV}$, it is clearly not possible to associate its spectral weight only with the much narrower pseudogap region. Instead, this low-energy peak most likely covers all unoccu-
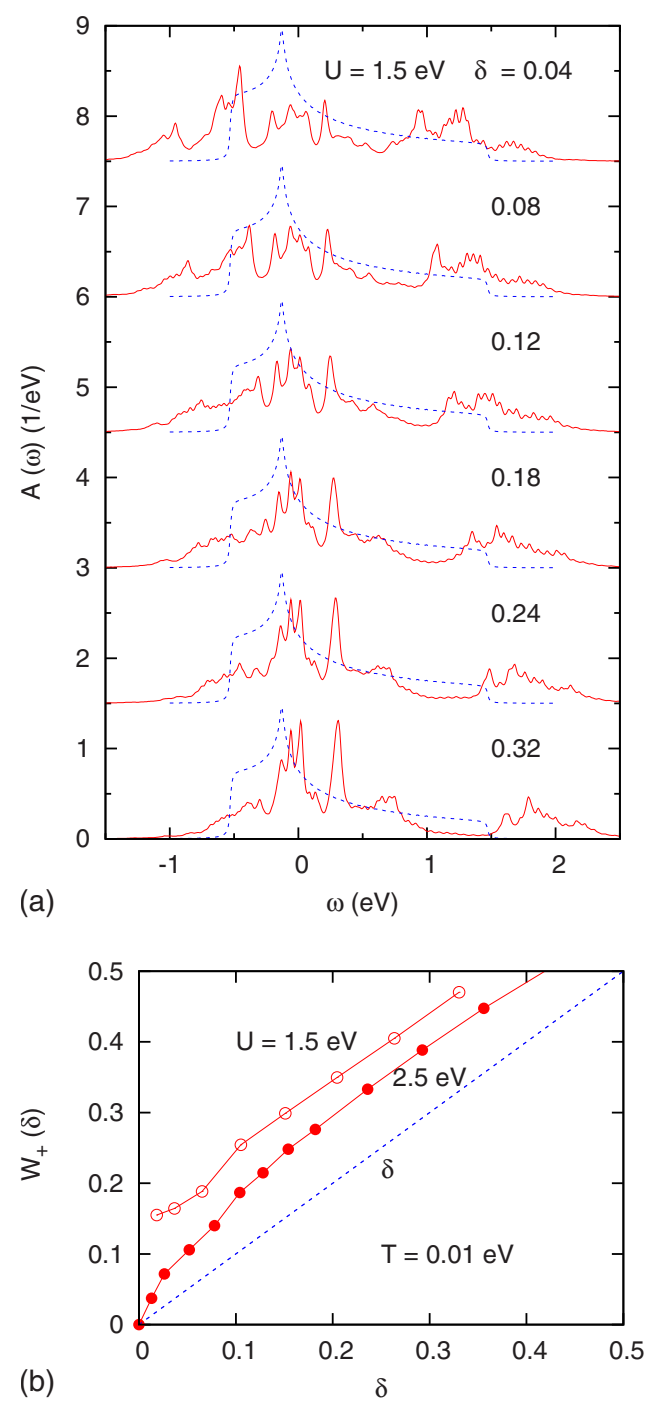

FIG. 4. (Color online) (a) Spectral distributions calculated within cluster DMFT for two-dimensional Hubbard model at several hole dopings (broadening $\gamma=0.02 \mathrm{eV}, U=1.5 \mathrm{eV}$, and $T$ $=0.01 \mathrm{eV}$ ). The dashed lines denote the bare density of states. (b) Integrated spectral weight of low-energy states per spin as a function of hole doping. The integration window extends to the dopingdependent minimum below the upper Hubbard band. Empty dots: $U=1.5 \mathrm{eV}$. Solid dots: $U=2.5 \mathrm{eV}$. 
pied states below the upper Hubbard band. The fact that this peak saturates near $\delta \approx 0.2$ in the experiment is therefore in conflict with the cluster DMFT results for the single-band Hubbard model shown in Fig. 3(a). According to the results provided in Fig. 3(b), the saturation obtained by Phillips and Jarrell $^{3}$ appears to be related to the small, dopingindependent cutoff energy. Since their spectra exhibit a similar doping variation as the ones shown here in Figs. 1 and 2, a larger doping-dependent cutoff energy should give an integrated low-energy spectral weight in agreement with the variation shown in Figs. 3(a) and 4(b).

As shown recently by Wang et al., ${ }^{12}$ the experimental data by Peets et al. are also not compatible with results of a single-site DMFT model calculation including $\mathrm{Cu} d$ and $\mathrm{O} p$ orbitals. It would be very interesting to generalize this model to allow for intersite correlations.

\section{CONCLUSION}

The integrated spectral weight of states induced in the Mott gap of the two-dimensional single-band Hubbard model via hole doping is examined within cluster DMFT based on finite-temperature exact diagonalization. The doping variation of this weight is shown to depend sensitively on the energy window in which the low-energy states are counted. If the cutoff energy is chosen to vary with doping and to lie in the minimum below the upper Hubbard band, qualitative agreement with model predictions by Eskes et al. is found. On the other hand, if the cutoff energy is taken to be independent of doping, the integrated spectral weight exhibits a completely different variation with doping. In particular, at small cutoff energies, approximate saturation at low doping is found. Since the XAS data by Peets et al. require integration over all unoccupied states except for the upper Hubbard band, we conclude that these data are not compatible with present cluster DMFT predictions for the single-band Hubbard model.

Note added in proof. As now pointed out by Phillips and Jarrell, the experimental data of Refs. 2 and 11 are in excellent agreement with the trend shown here in Fig. 3(a), with the exception of two data points at 0.3 hole doping. Thus, if the error bars of these points are sufficiently large, then there is no conflict between the data and the predictions of the single-band Hubbard model. ${ }^{13}$

\section{ACKNOWLEDGMENTS}

I would like to thank George Sawatzky for sending the preprint (Ref. 6) prior to publication and for numerous discussions. I would also like to thank André-Marie Tremblay for stimulating discussions.
${ }^{1}$ H. Eskes, M. B. J. Meinders, and G. A. Sawatzky, Phys. Rev. Lett. 67, 1035 (1991); M. B. J. Meinders, H. Eskes, and G. A. Sawatzky, Phys. Rev. B 48, 3916 (1993).

${ }^{2}$ D. C. Peets, D. G. Hawthorn, K. M. Shen, Y.-J. Kim, D. S. Ellis, H. Zhang, S. Komiya, Y. Ando, G. A. Sawatzky, R. Liang, D. A. Bonn, and W. N. Hardy, Phys. Rev. Lett. 103, 087402 (2009).

${ }^{3}$ P. Phillips and M. Jarrell, arXiv:1003.3412 (unpublished).

${ }^{4}$ M. H. Hettler, A. N. Tahvildar-Zadeh, M. Jarrell, T. Pruschke, and H. R. Krishnamurthy, Phys. Rev. B 58, R7475 (1998).

${ }^{5}$ M. Jarrell, Th. Maier, M. H. Hettler, and A. N. Tahvildarzadeh, Europhys. Lett. 56, 563 (2001); M. Jarrell, Th. Maier, C. Huscroft, and S. Moukouri, Phys. Rev. B 64, 195130 (2001).

${ }^{6}$ The same conclusion was reached independently by D. C. Peets, D. G. Hawthorn, K. M. Shen, G. A. Sawatzky, R. Liang, D. A. Bonn, and W. N. Hardy, arXiv:1004.1146 (unpublished).
${ }^{7}$ A. Georges, G. Kotliar, W. Krauth, and M. J. Rozenberg, Rev. Mod. Phys. 68, 13 (1996).

${ }^{8}$ G. Kotliar, S. Y. Savrasov, G. Palsson, and G. Biroli, Phys. Rev. Lett. 87, 186401 (2001).

${ }^{9}$ A. Liebsch and N.-H. Tong, Phys. Rev. B 80, 165126 (2009).

${ }^{10} \mathrm{~S}$. Sakai, Y. Motome, and M. Imada, Phys. Rev. Lett. 102, 056404 (2009).

${ }^{11}$ See also C. T. Chen, L. H. Tjeng, J. Kwo, H. L. Kao, P. Rudolf, F. Sette, and R. M. Fleming, Phys. Rev. Lett. 68, 2543 (1992); N. Nücker, E. Pellegrin, P. Schweiss, J. Fink, S. L. Molodtsov, C. T. Simmons, G. Kaindl, W. Frentrup, A. Erb, and G. MüllerVogt, Phys. Rev. B 51, 8529 (1995).

${ }^{12}$ X. Wang, L. de'Medici, and A. J. Millis, Phys. Rev. B 81, 094522 (2010).

${ }^{13} \mathrm{P}$. Phillips (private communication). 